\title{
High Intensity Broad Spectrum LEDs in the Near Infrared Range
}

\author{
József NÁDAS ${ }^{1, a^{*}}$, Vilmos RAKOVICS ${ }^{2, b}$ \\ 1 Óbuda University, Kandó Faculty of Electrical Engineering, Institute of Microelectronics and \\ Technology (Address: Tavaszmező u. 15. B. 014. 1084 Budapest, Hungary) \\ ${ }^{2}$ Hungarian Academy of Sciences, Centre for Energy Research, Institute of Technical Physics and \\ Materials Science (Address: Konkoly Thege M. út 29-33., 1121 Budapest, Hungary)

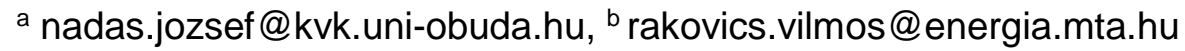 \\ ${ }^{*}$ corresponding author
}

Keywords: NIR LED, GalnAsP/InP LED, luminescence LED

\begin{abstract}
IR LEDs are widely used in the near-infrared (NIR) spectroscopy, especially in handheld devices for the detection and measurement of organic materials, allowing faster and cheaper examinations. The - $\mathrm{OH},-\mathrm{NH}$ and $-\mathrm{CH}$ functional groups found in organic substances can frequently be detected by spectroscopy through absorbance measurement at the resonance wavelength of valence-bond vibrations. The measured wavelengths are $4-2.5 \mu \mathrm{m}$, while signal to noise ratio of photon detectors is low due to thermal noise at room temperature. The 1st-3rd harmonic absorption bands are located in the range of the NIR, where smaller signals can be measured effectively in practice. The LEDs have tunable narrow wavelength range therefore they are suitable for such measurements as radiation sources. Further advantages of LEDs compared to incandescent lamps are small dimensions, high efficiency, and low power consumption. In order to tune the emission wavelength of the LED, the composition of the semiconductor light-emitting layer has to be properly set. The change in chemical composition will generally affect the lattice constant too. The crystal defects caused by lattice-mismatch often reduce the efficiency. The emission wavelength is tunable in the quaternary GaInAsP/InP material system meanwhile the lattice-constant remains unchanged. Absorption band of the organic materials is generally broader than the emission spectrum of the LED, therefore a broader emitter is preferred. To achieve this, a number of solutions are known. One of these is to use multiple layers with different composition, where the primary light comes from the active layer at the p-n junction of the diode, then excites the second smaller band gap layer producing longer wavelength secondary light by photoluminescence. The transmitted part of the primary light, together with the secondary light, results in a broader spectrum. This method is not yet implemented in this material system, so my work is focused mainly on this approach.
\end{abstract}

\section{Introduction}

Near infrared (NIR) spectroscopy is suitable to detect $-\mathrm{OH},-\mathrm{NH},-\mathrm{CH}$ functional groups in organic materials, with absorbing resonant wavelength of stretching vibrations typical of these bonds. The presence of organic materials and their concentration can be inferred from the detection of materialspecific functional groups. The bond vibration wavelengths are $\lambda=3-4 \mu \mathrm{m}$ in these functional groups, however, in practice, in orders of magnitude smaller signals can be effectively measured in the range of $1^{\text {st }}-3^{\text {rd }}$ harmonics $(\lambda=1000-1800 \mathrm{~nm})$ due to better signal to noise ratio of shorter wavelength detectors. Another advantage is that the short wavelength radiation penetrates deeper into the material, this way the volume composition of samples can be measured. The incandescent lamp was the typical traditional light source of NIR spectroscopy earlier. The maximum radiation of incandescent lamps is in this range 1000-1200 nm (depending on temperature of filament), but the wavelength range necessary for the measurement is narrow within the wide range of radiation. The useful radiation power compared to the power consumption of incandescent lamp is very small, the efficiency is poor. The LED as radiation source of NIR spectroscopy compared with the incandescent lamp has a lot of advantages [1]. The LED wavelength range is narrow and can be 
planned. The advantages of LEDs are their short response time $\left(\mathrm{x}^{*} 10^{-9} \mathrm{~s}\right)$, good focusability, high efficiency, low power consumption, and multiple expected lifetime compared to incandescent lamps [2]. The latter features of LED make it especially suitable for use in hand-held devices, allowing faster and less expensive measurement than before. Application areas are for example biological samples, human health diagnostics, measurements of agricultural crops, measurements in oil industry, environmental and plastics industry measurements. The disadvantage of LEDs comes from one of their advantageous features: the narrow range of radiation allows a specific wavelength measurement only. To detect or determine the concentration of a specific organic material measurements have to be taken at several (at least two) wavelengths, because they should be distinguished from other present substances in the environment (this usually means water or other organic materials). To achieve this, the operation of several (typically three) different wavelength LEDs is mostly common in current practice. The drawback of the solution is that the radiation source is not entirely focusable, and the temperature dependence and aging parameters of various wavelength emitting LEDs are different. The ideal source of radiation is a single semiconductor structure that emits a wide band range while it has minimum temperature dependence.

\section{Experimental}

Selection of materials. We had to make a special LED structure from compound semiconductor, therefore firstly we looked for suitable material system. The GaInAsP compound semiconductor is suitable as an efficient and accurate NIR radiation source.

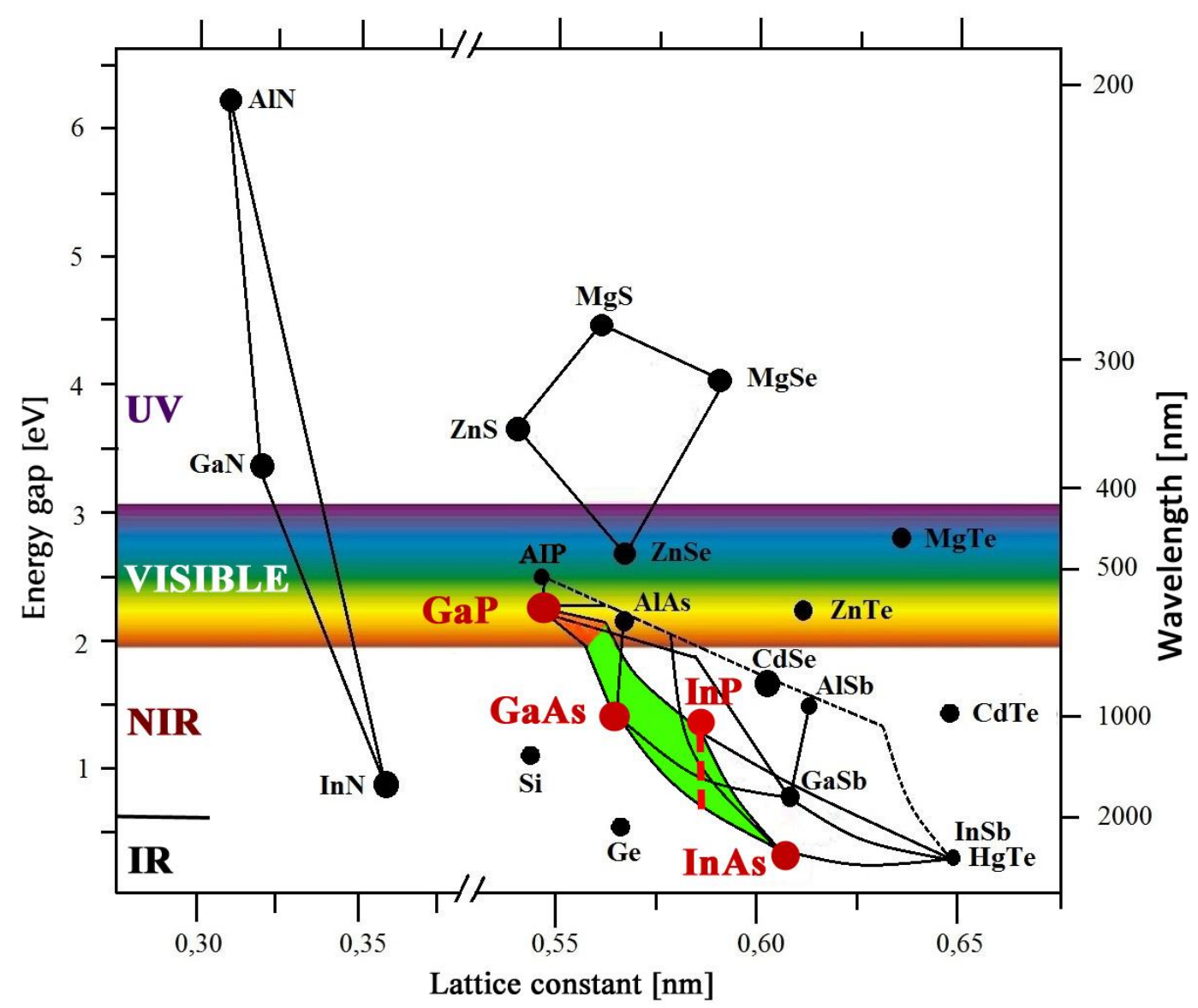

Fig. 1. Lattice constant - band gap relationship in compound semiconductors [4]. Along the vertical dashed line tunable wavelength NIR semiconductors lattice-matched to InP are made possible.

The wavelength range of compound semiconductor LED is narrow and it is tunable by changing the composition of the active layer, therefore it is suitable as a radiation source for NIR measurements. In order to tune the wavelength of the radiation the composition of the active layer has to be 
changed, however, the lattice constant usually changes along with it. The errors caused by latticemismatch usually reduce the efficiency of the devices. The wavelength is tunable in the GaInAsP/InP material system meanwhile the lattice-constant remains unchanged. GaInAsP latticematched to InP LED structure can be prepared in the 960-1670 nm wavelength range where the substrate absorption is negligible [3, 4].

LED technology and characterization. We were grown LEDs by liquid phase epitaxy (LPE) because it is an ideal method for growing thin layers, where thicknesses and composition must be tuned up accurately. On the other hand it is relatively cheap and easy, material-saving, relatively quick and typically fit to research purpose production. We made optical transmission measurements because these are sufficient for prediction of the peak emission wavelength of LED, as the first derivative of the transmission spectrum of the wafer shows the same maximum as the emission peak of the device prepared from the wafer. After preparation our LED characteristics were measured as a function of driving current and temperature. As temperature increases, the peak of the emission spectrum shifts towards higher wavelengths, while the efficiency is decreasing. The peak wavelength shows a slight blue shift at high current, but this does not compensate the heatinduced red shift, only reduces it at real operating conditions [5]. Our goal was minimizing current and temperature dependence of the LED emission spectrum.

Possible broad spectrum LED structures. The absorption bands of organic materials are generally wider than the bandwidth of a single LED therefore a wider wavelength range of operation is preferred. To achieve this, there are several known solutions, e.g. application of phosphor, a group of several different wavelength LEDs, and other structural architectures (e.g. tandem LED). We have prepared multiple layers of different composition, where the light originated from active layer exits the LED unchanged only partially, meanwhile remainder radiation excites additional layer(s), and realizes several radiation peaks with luminescence. Structures with luminescent layer are called Photon Recycling Semiconductor (PRS) structures [6, 7].
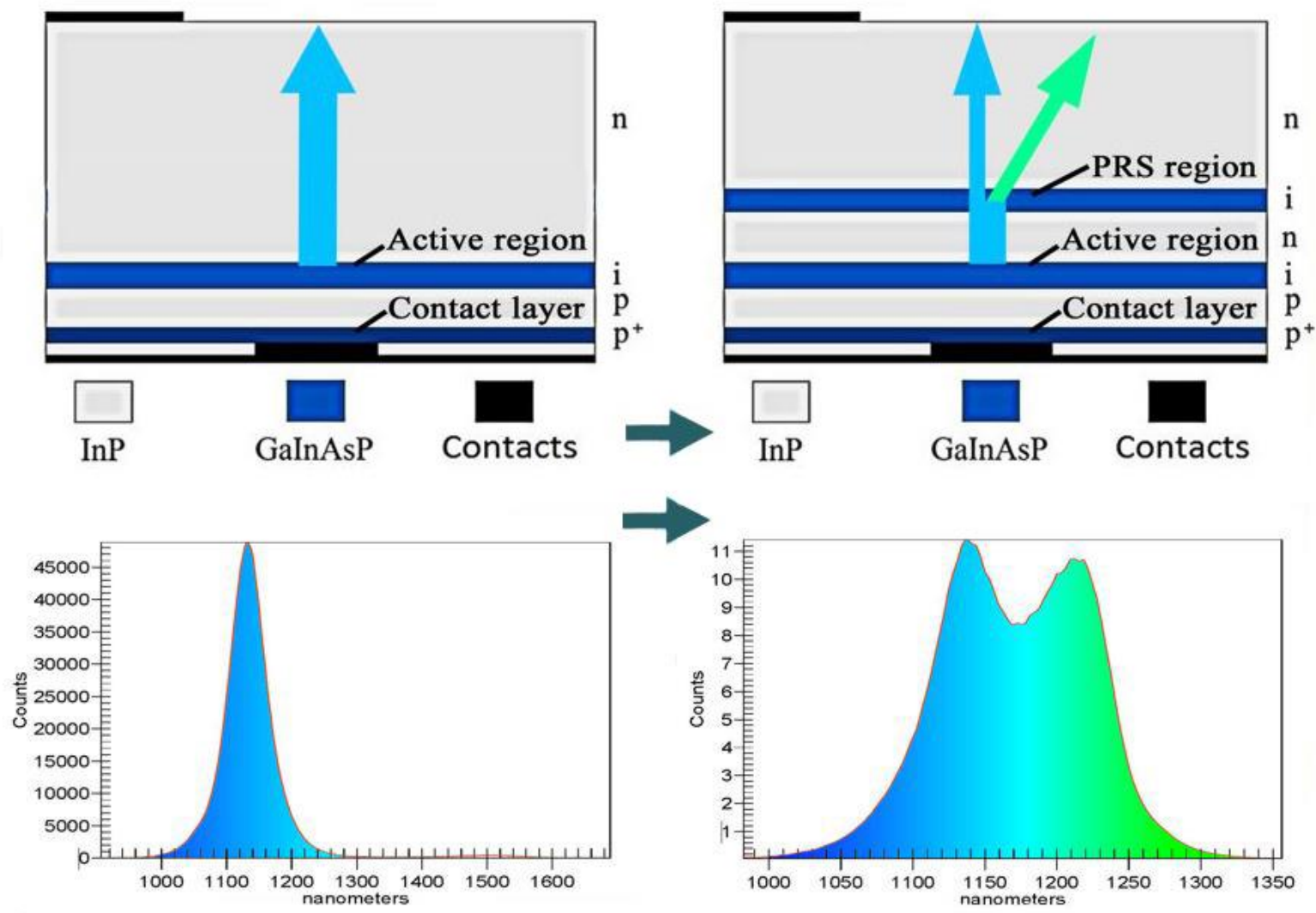

Fig. 2. Ordinary layer stucture and spectra of InGaAsP/InP LED (left) and layer structure including a luminescent layer with widened spectra (right). 
We compared our $1120 \mathrm{~nm}$ ordinally LED and 1120-1220 nm single chip luminescent LED in Fig. 2. Luminescent LEDs are capable of emitting light in wide wavelength range, so they can find application as light sources for modern detector diode array spectrometers.

Temperature sensitivity of the LED spectrum. The intensity of LED emission at different part of the spectrum may vary in opposite direction due to changing the operation temperature. This could result in measurement errors which is difficult to correct $[8,9]$. The shift of the emission spectrum of multi wavelength LEDs is a sum the change of the emission spectra of its components.

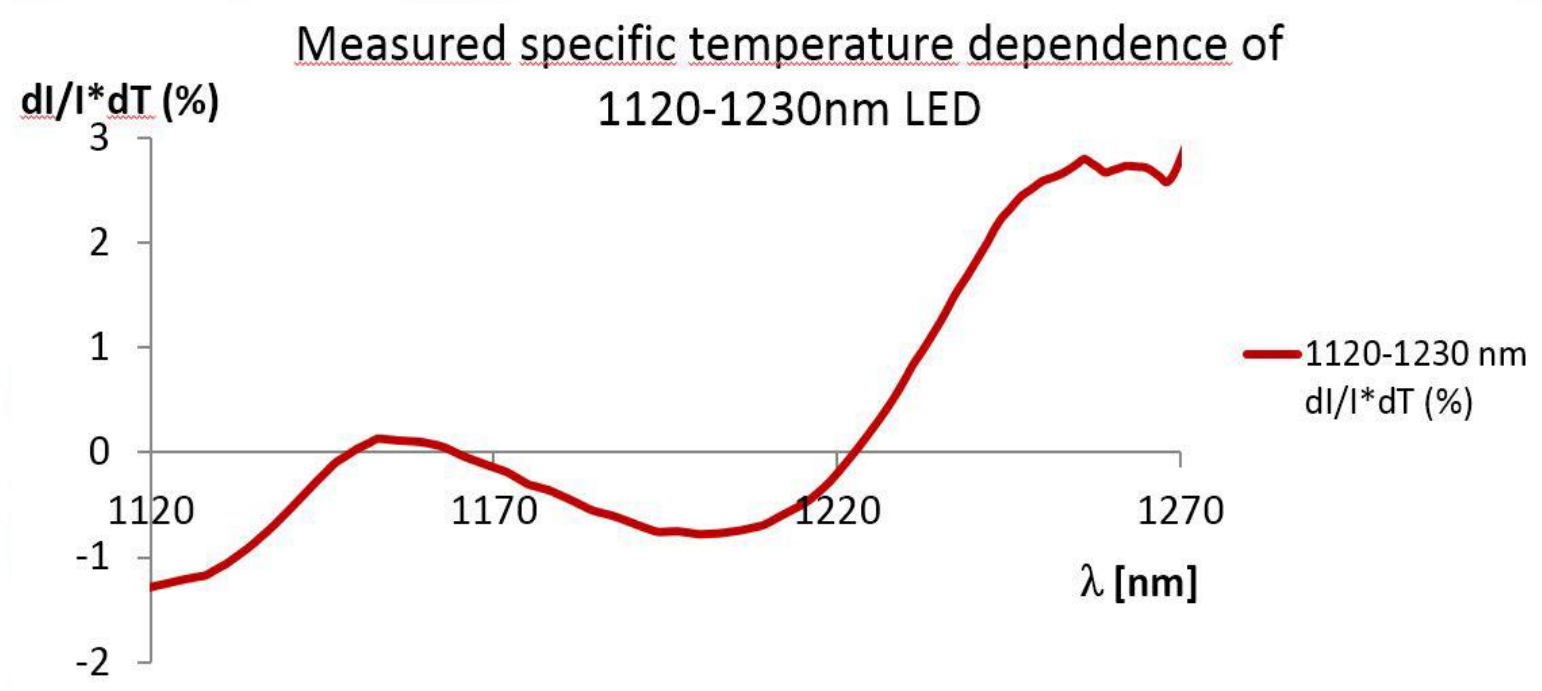

Fig. 3. Specific temperature dependence of radiation intensity of LED with 1120-1230 nm radiation peaks. This experimental LED were grown by ourselves. As we expected, almost temperature independent section is formed between closely radiation peaks with nearly same amplitude.

Temperature dependence of the spectrum can be design in two ways. Important sections for a given measurement points can have the same temperature coefficients, ore can have near zero between the two adjacent peeks of the LED. Owing to this, another possible area of use is application to meet the needs of small temperature-dependent use, especially in handheld instrumental measurements, where complex circuit correction cannot be realized because of simplicity, or temperature correction because of low power consumption.

Design of InGaAsP/InP NIR LED for measurement of ethanol-water mixture. Ethanol is a good model material which represents organic materials well in measurements for water-ethanol (or other organic materials) systems. Detection of $-\mathrm{OH}$ and $-\mathrm{CH}$ bonds of human tissues and organic plant materials in water is very similar. Measurable optical transmission thus small sample thickness is required for accurate determination of the composition of the samples. Relative optical transmission of ethanol to water has its maximum around $1400 \mathrm{~nm}$ wavelength where difference of absorption at the sharp edge is significant between these materials [1]. It is not enough to measure the optical transmission only at one wavelength in the case of determining concentration of ethanol from a three-component mixture. Measurements are taken where the optical absorption of the two component are the same $\left(\lambda_{1}=1200 \mathrm{~nm}\right)$, at ethanol minimum $\left(\lambda_{2}=1300 \mathrm{~nm}\right)$ and water maximum $\left(\lambda_{3}=1450 \mathrm{~nm}\right)$. Measurements at $\lambda_{1}$ and $\lambda_{3}$ are sufficient if ethanol-water mixture contains these two components only. The intensity of transmitted light can be evaluated till $1400 \mathrm{~nm}$ therefore modifying $\lambda_{3}$ to $\lambda_{3}{ }^{\prime}=1400 \mathrm{~nm}$ is appropriated. We were grown luminescent LED had radiation peaks at $\lambda_{1}=1200 \mathrm{~nm}$ and $\lambda_{3}{ }^{\prime}=1400 \mathrm{~nm}$. Temperature dependence of 1200-1400 nm LED grown for measurement of ethanol-water mixture can be corrected linearly at peak wavelengths. Temperature independent sections are created above peak wavelengths of 20-50 nm. These sections are particularly suitable for measuring if there is proper amplitude and detector sensitivity. To do so, 
the wavelengths of LED should be tuned so that these wavelength values will set to be on sections of measurement.

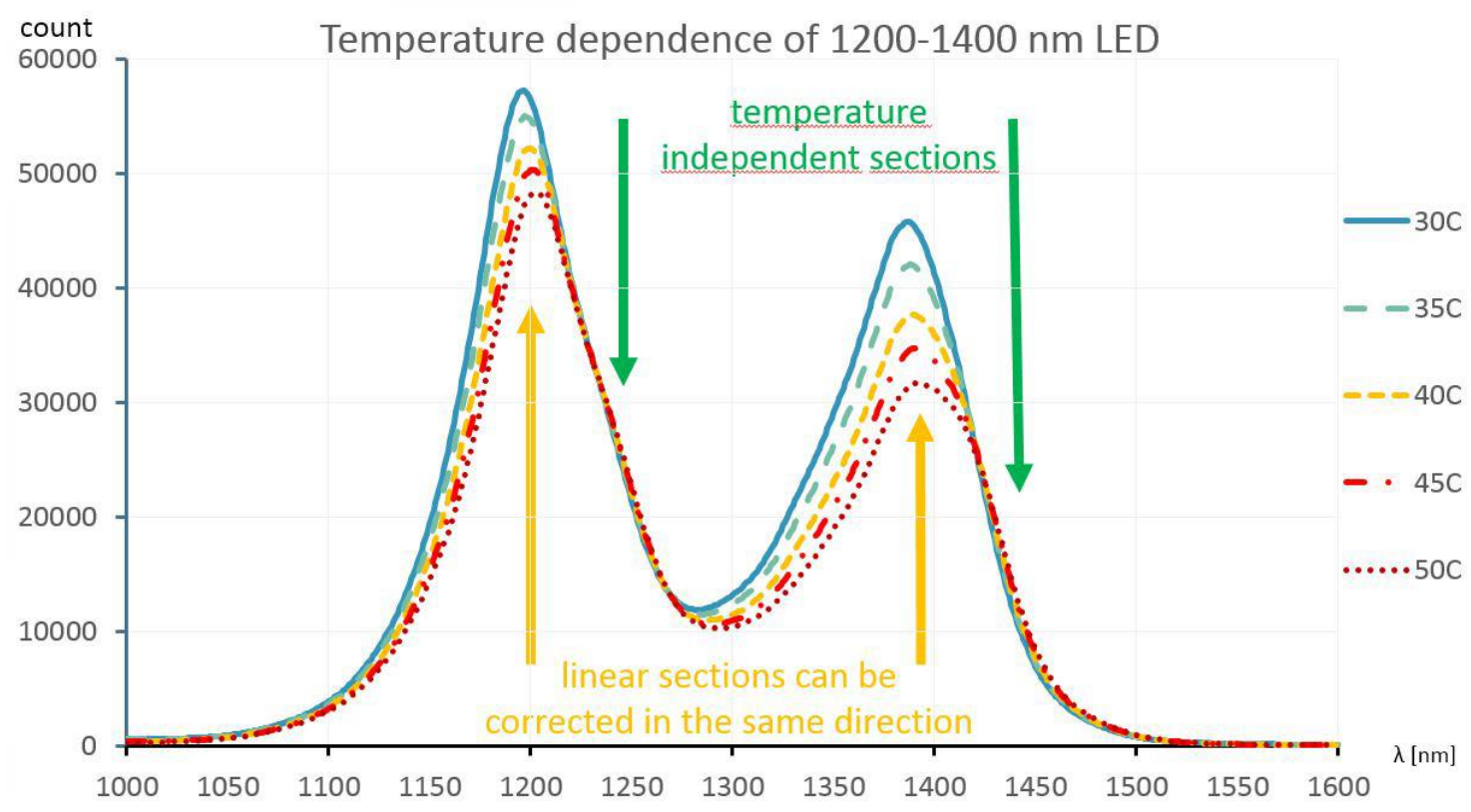

Fig. 4. Temperature dependence of 1200-1400 nm LED grown by us for measurement of waterethanol mixture.

Growth conditions. Each of the four components (Ga, In, As, P) are relatively abundant in the luminescent layer tuned to a wavelength of $1400 \mathrm{~nm}$. The miscibility gap is crucial in determination of growth temperature. To avoid phase separation, growth temperature we kept higher, $645^{\circ} \mathrm{C}$ at the upper limit of the usual $600-650^{\circ} \mathrm{C}$. Luminescent layer is less sensitive to growth mismatch. We were grow an anti-meltback layer with composition tuned for $1200 \mathrm{~nm}$ (drawn by hatched pattern in Fig. 5.). It is necessary after growing luminescent layer.

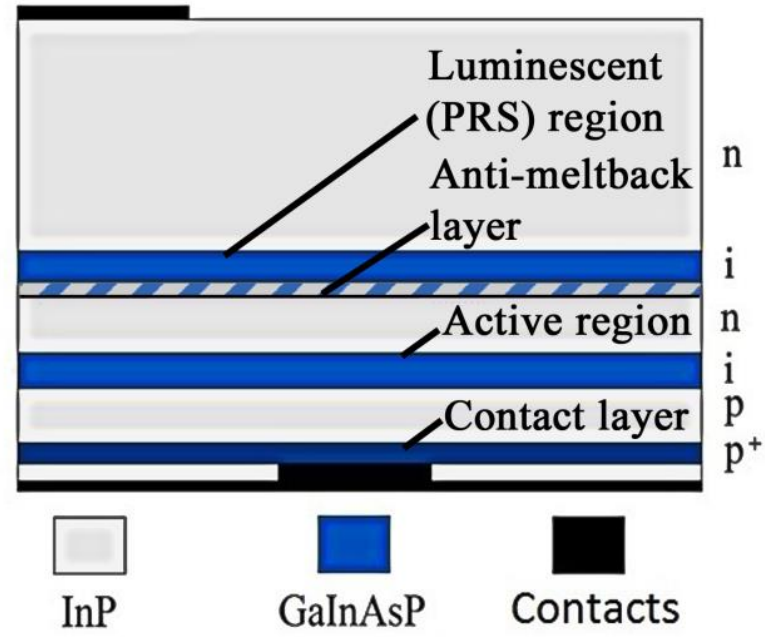

Fig. 5. The layer structure of broad spectrum GaInAsP/InP NIR LED.

\section{Conclusions}

GaInAsP/InP LEDs with spatially and spectrally stabile broad emission spectrum can be prepared in a single semiconductor structure. The LED structure contains an electrically pumped active layer at p-n junction as a primary light source, and a wavelength converter region required for broadening 
the spectrum of the device by luminescence. The wavelength of the active and luminescent layer(s) and their intensity is tunable with material composition, and the layer thicknesses. This allows to grow LEDs which are efficient, precisely tuned to the desired wavelength in wide range, narrow beam and almost independent of temperature. Two-wavelength LED (1200 and $1400 \mathrm{~nm}$ ) for ethanol in water measurements was developed and characterized. It was shown that growth of an anti-meltback layer on the luminescent layer is necessary for good device parameters. The device structure has closely spaced emitting layers and small emitting area. As a result of the compact structure the devices have good temperature stability and high brightness.

\section{Summary}

GaInAsP/InP LED structures with multiple wavelengths were grown with LPE method. The emission spectra of these devices can be adjusted according to the specific application of NIR spectroscopy. The emission of the LEDs needs to be broadened in a new way so that it keeps the focusability, almost independent of temperature, accurately tuned to a desired wavelength range and the spectral intensity is adjustable. The wavelength of the active and luminescent layer is tunable with material composition and the amplitudes with the layer thicknesses in LEDs emitting two wavelengths. A two wavelength single chip LED was developed for optical ethanol concentration measurements in water solution. We optimized the layer structure and the device technology according to the requirements of the application. The spectrum of LED fits the absorption maximum of O-H and C-H bonds in NIR range. We demonstrated the benefits of the new NIR light source by IR transmission measurements in ethanol-water system.

\section{References}

[1] Rakovics V., Réti I.: Infravörös diódák alkalmazása az élelmiszerek spektroszkópiai vizsgálatára in: Műszaki Kémiai Napok '08, 2008. április 22-24. Veszprém, Hungary, pp. 64-68.

[2] Zarr, R.: LEDs Line Up To Replace Residential Incandescent Bulbs in: Electronic Design, Vol.02/2013, pp. 14-15.

[3] Réti I.-Ürmös A.-Nádas J.-Rakovics V.: Nanostruktúrás LED-ek in: Elektrotechnika 2014/11 pp. 19-21.

[4] E. Kuphal: Phase Diagrams of InGaAsP, InGaAs and InP Lattice-Matched to (100) InP in: Journal of Crystal Growth 67 (Amsterdam, 1984) pp. 441-457.

[5] Rakovics V.-Nádas J.-Réti I.-Dücső Cs.-Battistig G.: Broad spectrum GaInAsP/InP near infrared emitting device, Poster in section TOP8 the 23rd HETECH 2014 Conference 1215.10.2014 Justus Liebig University Giessen, Germany.

[6] Rakovics V.: Optical investigation of InGaAsP/InP double heterostructure wafers, Advanced Optoelectronics and Lasers (CAOL), 2010 International Conference on, Sevastopol, Ukraine, 2010.09.10-2010.09.14. IEEE Communications, 2010. pp. 216-218.

[7] Rakovics V, Balázs J, Réti I, Püspöki S, Lábadi Z.: Near-Infrared Transmission Measurements on InGaAsP/InP LED Wafers, Physica Status Solidi C-Conferences and Critical Reviews 00: (2003) (3) pp. 956-960.

[8] Rakovics V, Püspöki S, Balázs J, Réti I, Frigeri C.: Spectral characteristics of InP/InGaAsP Infrared Emitting Diodes grown by LPE Materials Science and Engineering B - Solid State Materials for Advanced Technology 91-92 (2002), pp. 491-494.

[9] Rakovics V, Balázs J, Püspöki S, Frigeri C.: Influence of LPE growth conditions on the electroluminescence properties of $\operatorname{InP} / \operatorname{InGaAs}(\mathrm{P})$ infrared emitting diodes Materials Science and Engineering B - Solid State Materials for Advanced Technology 80: (1-3) (2001), pp. 18-22. 